\title{
Autonomous Landing and Hazard Avoidance Technology (ALHAT)
}

\author{
Dr. Chirold Epp \\ ALHAT Project Manager \\ Johnson Space Center
}




\section{OUTLINE}

- ALHAT BACKGROUND

- ALHAT VISION STATEMENT

- ALHAT MISSION PHASES

- TERMINAL DESCENT PHASE

- SAFE AND PRECISE LANDING

- LIGHTING

- LANDER TOLERANCE

- HDA TERRAIN SENSORS

- HDA SENSOR PERFORMANCE

- CREWED LANDINGS

- SUMMARY 
- In October 2005, the Exploration Systems Mission Directorate at NASA Headquarters assigned the development of new technologies to support the return to the moon.

- One of these was Autonomous Precision Landing and Hazard Detection and Avoidance Technology now known as ALHAT

- ALHAT is a lunar descent and landing GNC technology development project led by Johnson Space Center (JSC) with team members from Langley Research Center (LaRC), Jet Propulsion Laboratory (JPL), Draper Laboratories (CSDL) and the Applied Physics Laboratory $(\mathrm{APL})$ 


\section{ALHAT VISION STATEMENT}

Autonomous Landing Hazard Avoidance Technology (ALHAT)

- Develop and mature to TRL 6 an autonomous lunar landing GN\&C and sensing system for crewed, cargo, and robotic lunar descent vehicles. The System will be capable of identifying and avoiding surface hazards to enable a safe precision landing to within tens of meters of certified and designated landing sites anywhere on the Moon under any lighting conditions.
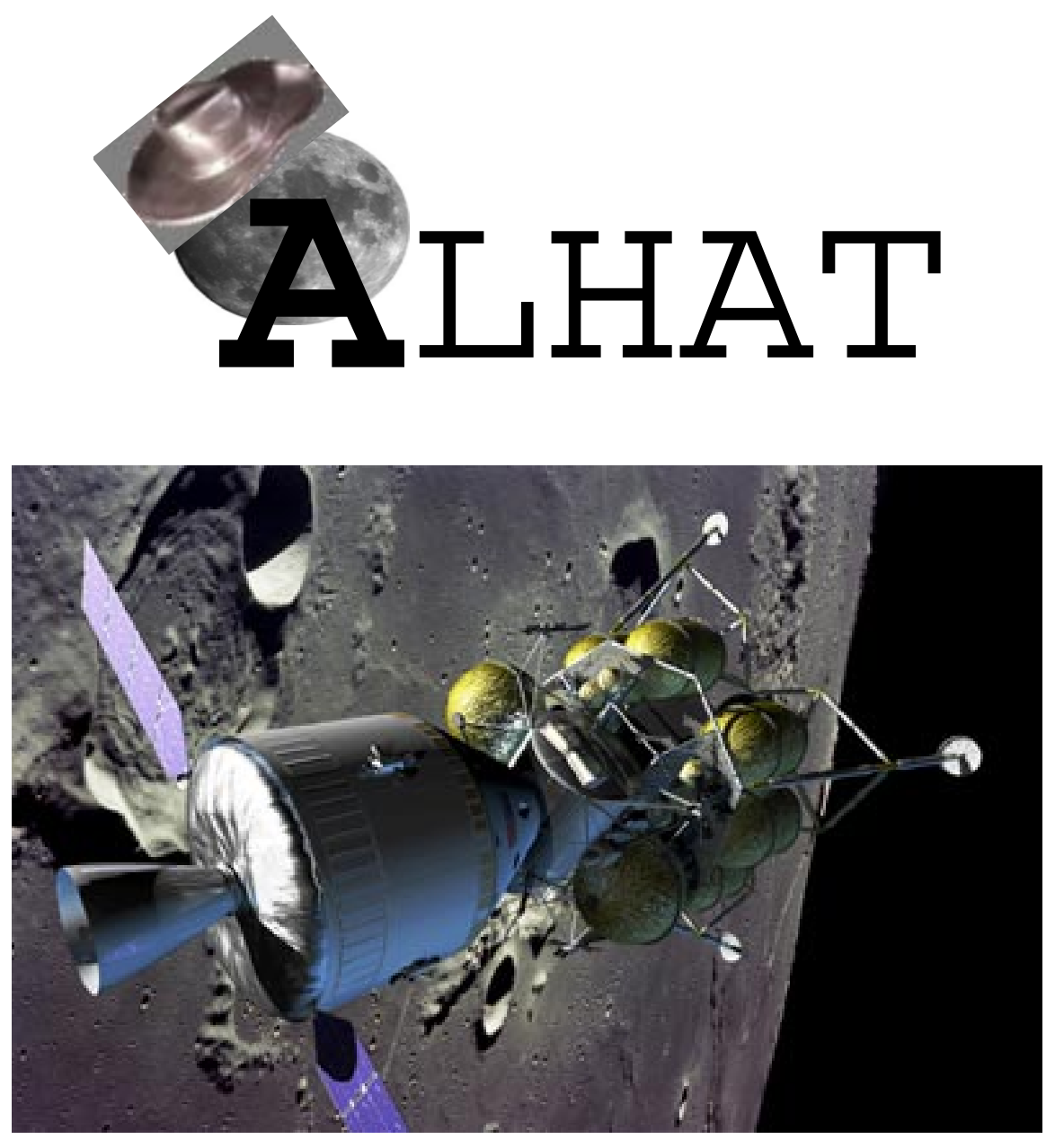


\section{ALHAT MISSION PHASES}

Autonomous Landing Hazard Avoidance Technology (ALHAT)

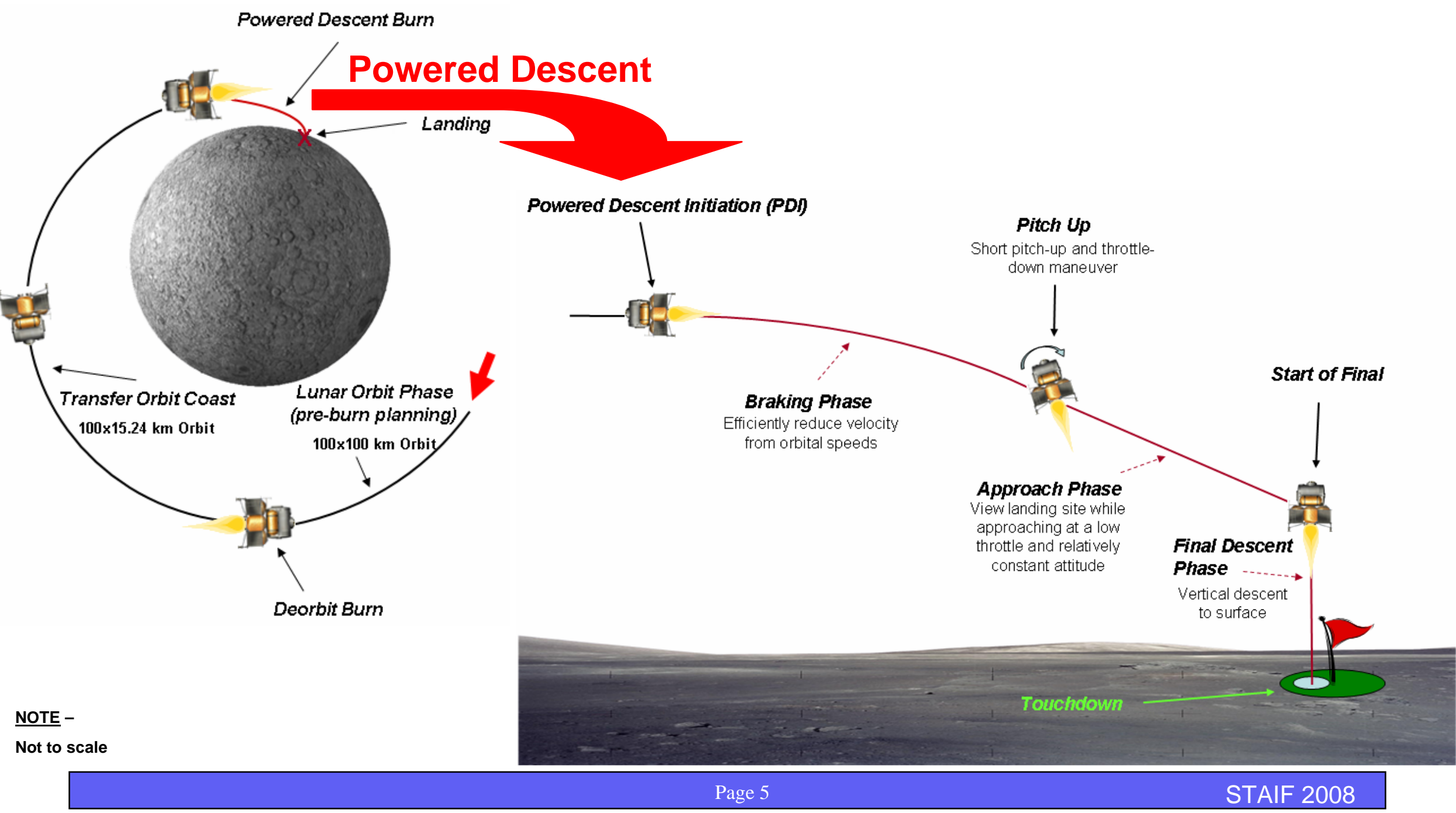




\section{TERMINAL DESCENT PHASE}

- PDI maneuver guidance targets an altitude and velocity at approximately $1.5 \mathrm{~km}$ from the landing site to execute the pitch-up maneuver and begin the final phase of landing with crew viewing out the window

- This phase requires hazard knowledge and the ability to identify a safe landing site

- ALHAT assumes no a priori knowledge of surface hazards

- Unclear what hazard resolution to expect from LRO but definitely will not have a priori surface hazard knowledge globally

- Most challenging and difficult landing phase

- Most important information is hazard elevation relative to the surrounding surface (above or below)

- Also important to maintain a good surface relative navigation state during this phase

- Following slides discuss major factors affecting this phase 


\section{SAFE AND PRECISE LANDING}

- The ALHAT project has accepted the challenge of global access to the moon

- Global access is interpreted to mean any terrain where there exists an area certified for landing within the tolerance of the lander

- Implies any lighting conditions because of places like the south pole where the maximum sun elevation angle is $1.5^{\circ}$

- Requires real-time hazard information

- Requires real-time surface relative navigation to land relative to a surface feature in a safe hazard-free area 
- CA0046-PO The Constellation Architecture shall conduct Lunar Sortie missions so that surface stays are conducted during periods of lunar daylight. [ARDIG]

- Not a specific landing lighting requirement but could be interpreted to mean no dark landings

- If the landing destination is Shackleton area this could mean landing with sun elevation angle of $0.5^{\circ}$ and launching from the surface with sun elevation angle of $0.5^{\circ}$ for a 7 day stay

- Daylight and what one can see with the human eye or cameras is an open question for places like Shackleton Crater where daylight has a sun elevation angle of $-1.5^{\circ}$ to +1.5 degrees.

- Opinions vary.

- Apollo requirements were for the sun to have a view angle that differed from the sun angle by more than $2^{\circ}$ in both elevation and azimuth 


\section{LIGHTING}

Autonomous Landing Hazard Avoidance Technology (ALHAT)

Kaguya North Pole Image

$1.5^{\circ}$ sun elevation

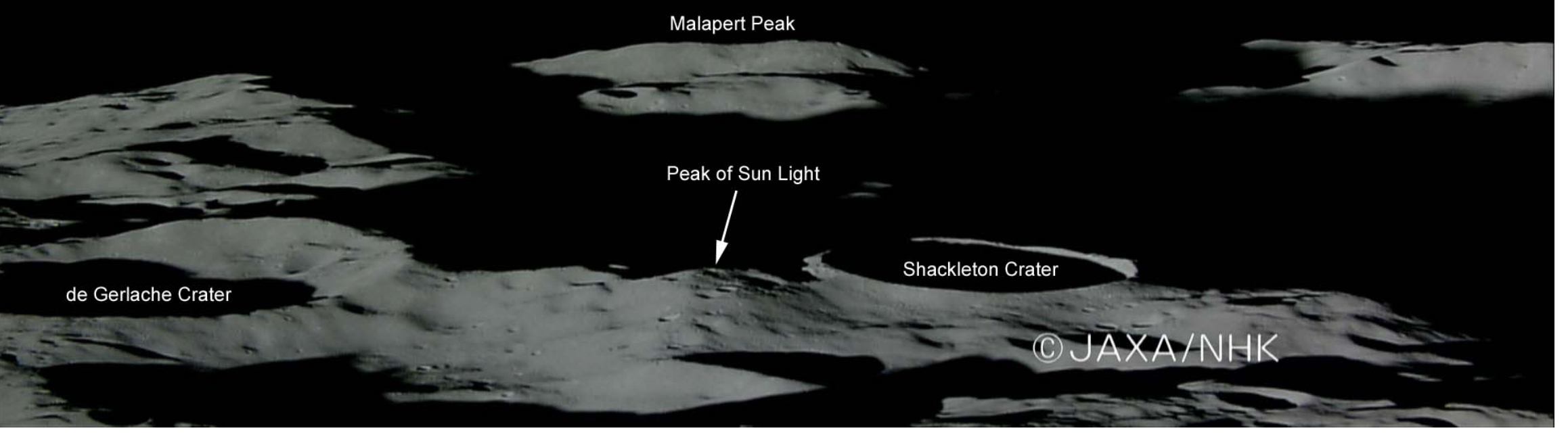




\section{LANDER TOLERANCE}

Autonomous Landing Hazard Avoidance Technology (ALHAT)

Some hazards recognizable during pre-flight planning....

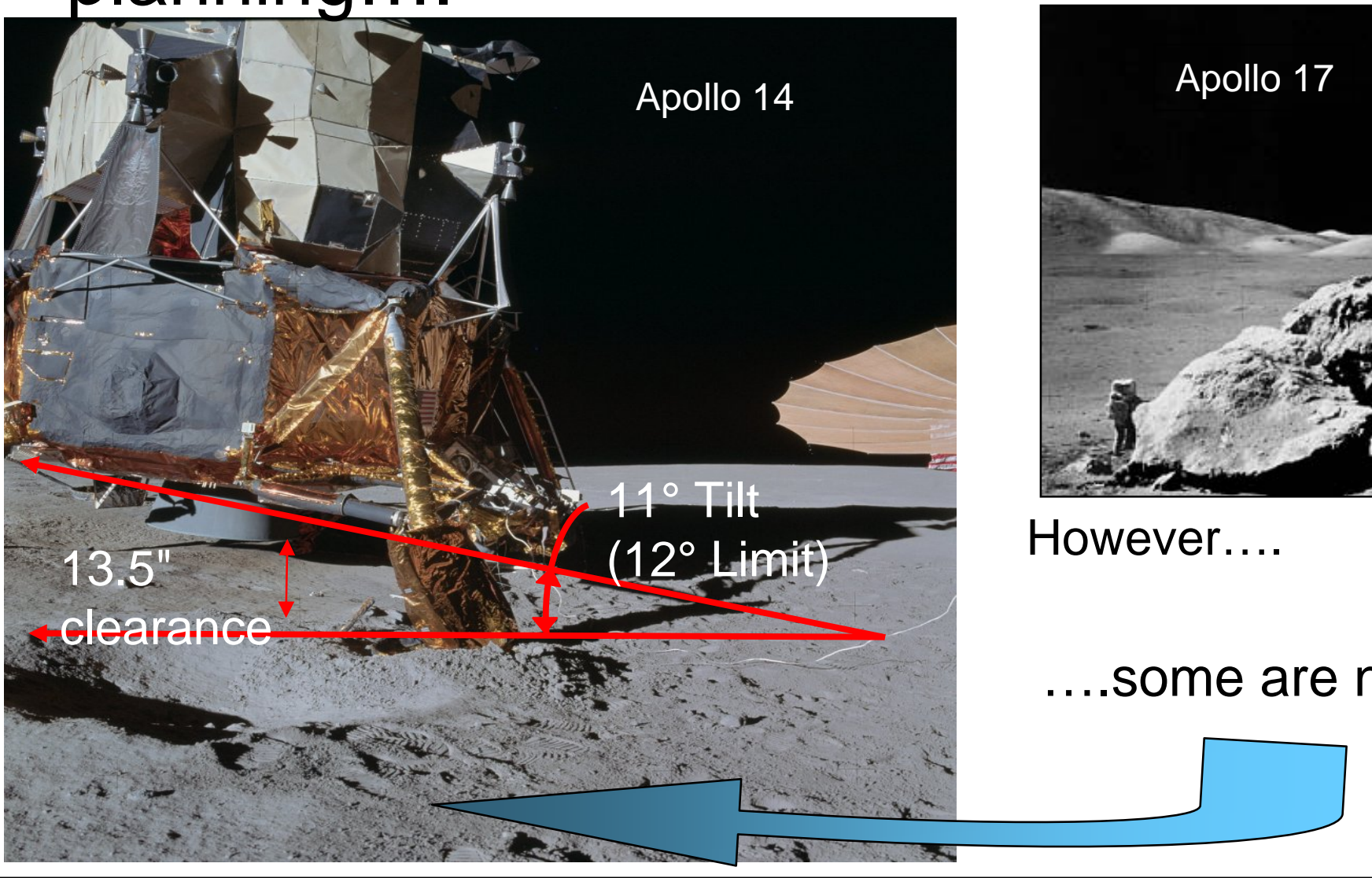




\section{LANDER TOLERANCE}

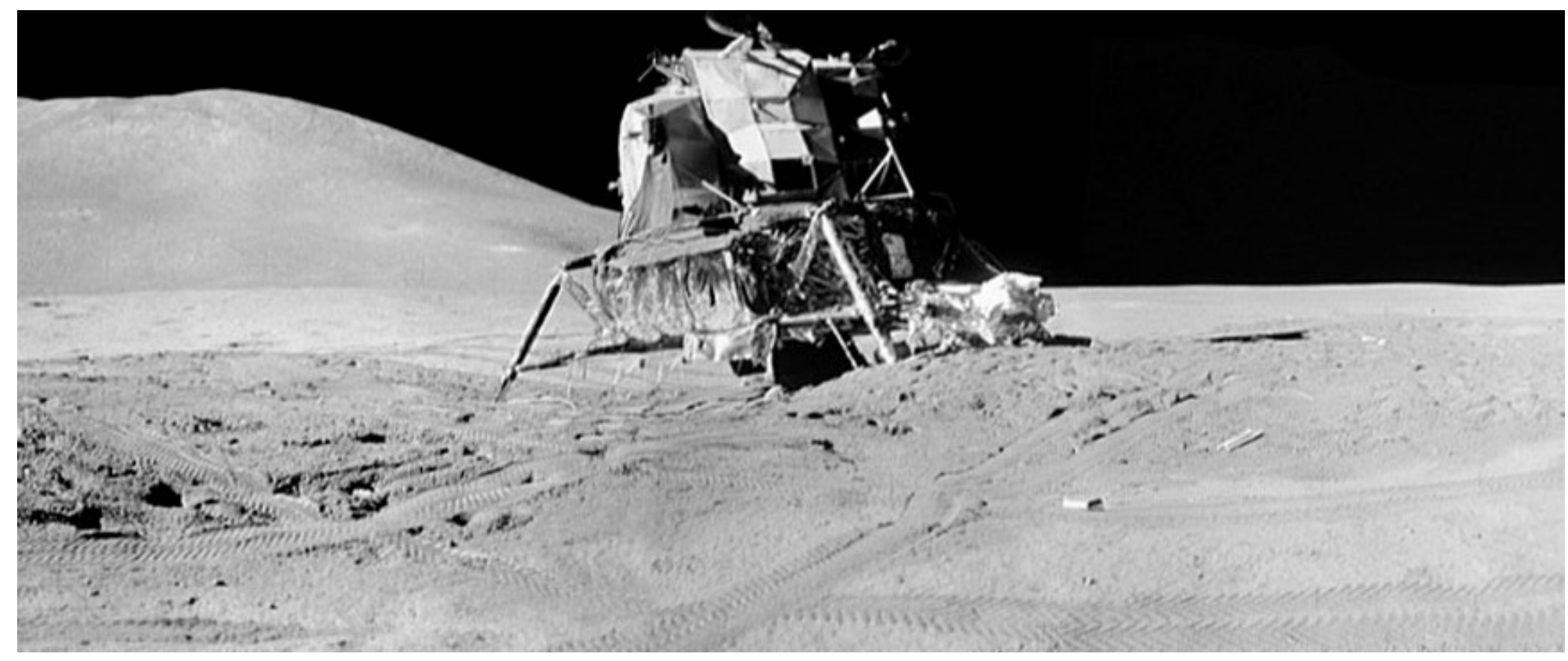

Apollo 15 set down on the rim of a small crater, damaging the engine bell and tilting at $\sim 10^{\circ}$ 
- Apollo limit was to tolerate rocks, holes and slopes that did not produce a vehicle that was more than $12^{\circ}$ from the vertical

- Vehicle footprint and this kind of constraint defines the hazards that need to be tolerated

- ALHAT is using very conservative requirements for mininum detectable hazards until something definite is given

- $30 \mathrm{~cm}$ hazards (rocks and holes) and $5^{\circ}$ slopes

- If the ALHAT system can work for these requirements it will work for less conservative requirements

- The probability of finding a safe site improves rapidly with more tolerant requirements 


\section{LANDER TOLERANCE}

Autonomous Landing Hazard Avoidance Technology (ALHAT)

Probability of finding one safe landing area

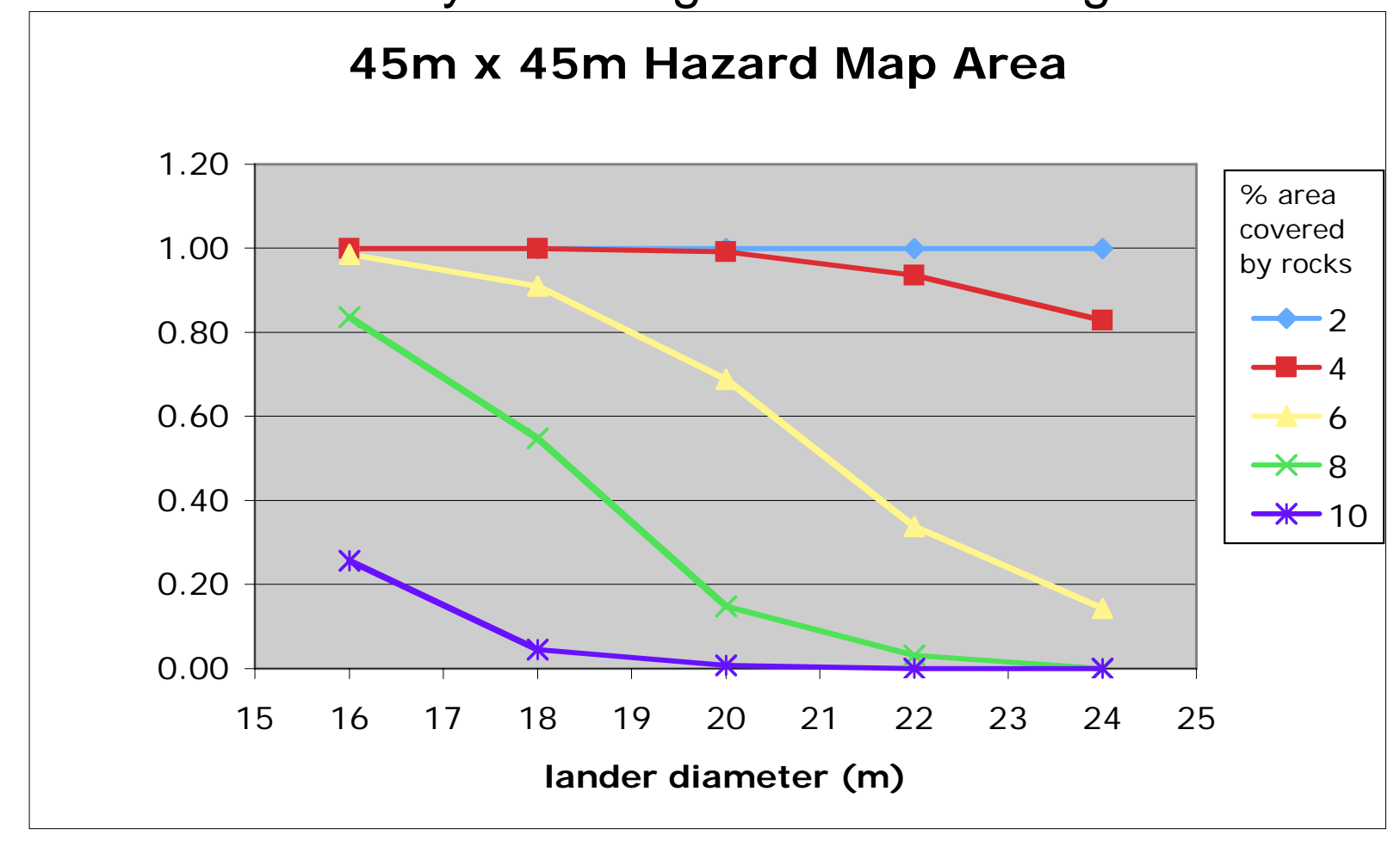

- Only rocks considered based on published statistical rock distribution models

- $30 \mathrm{~cm}$ rock is considered a hazard

Monte Carlo Analysis by Dr. Andrew Johnson, JPL, 2007 


\section{LANDER TOLERANCE}

Autonomous Landing Hazard Avoidance Technology (ALHAT)

Probability of finding one safe landing area

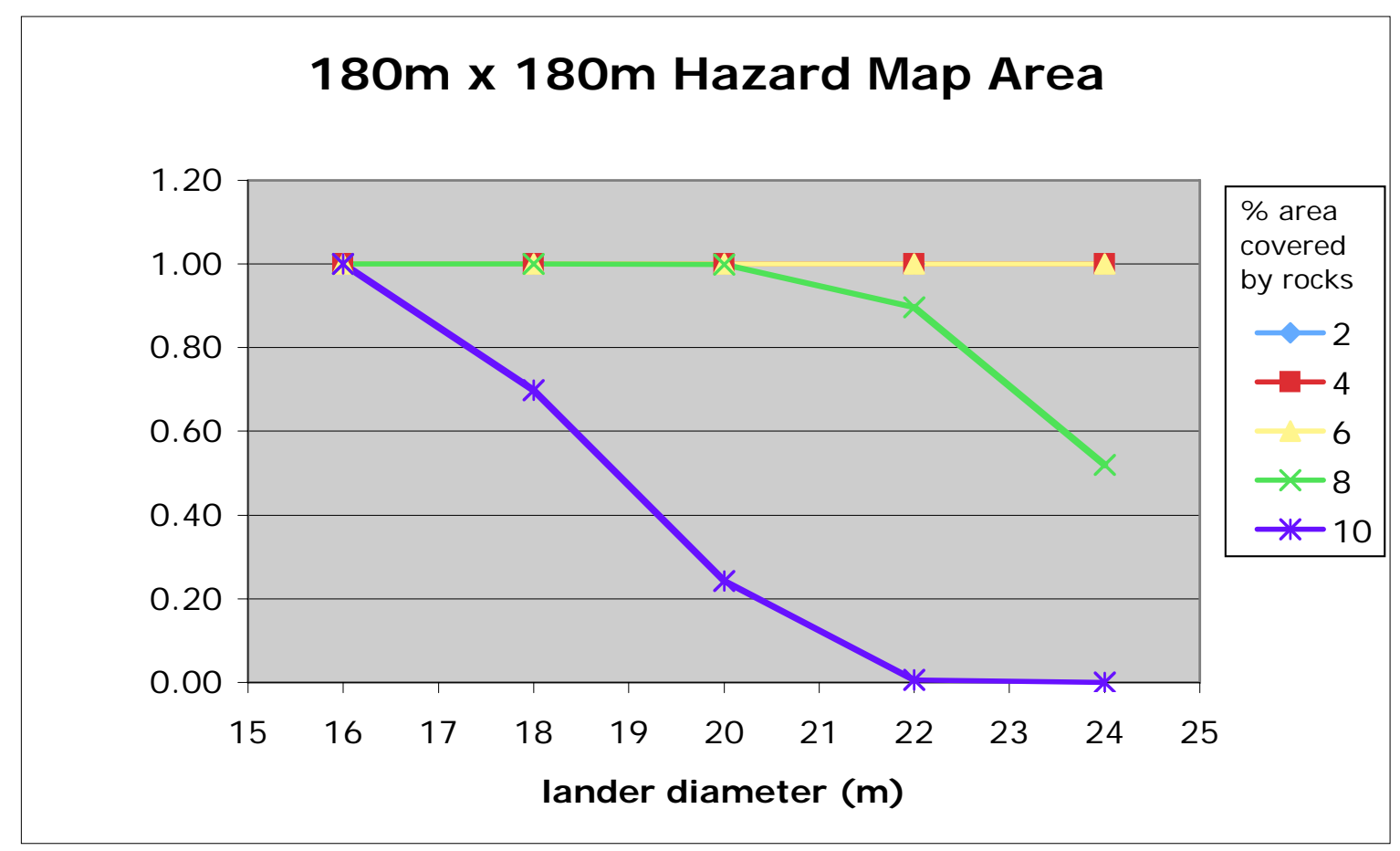

- Only rocks considered based on published statistical rock distribution models

- $30 \mathrm{~cm}$ rock is considered a hazard

Monte Carlo Analysis by Dr. Andrew Johnson, JPL, 2007 


\section{LANDER TOLERANCE}

Autonomous Landing Hazard Avoidance Technology (ALHAT)

Probability of finding one safe landing area

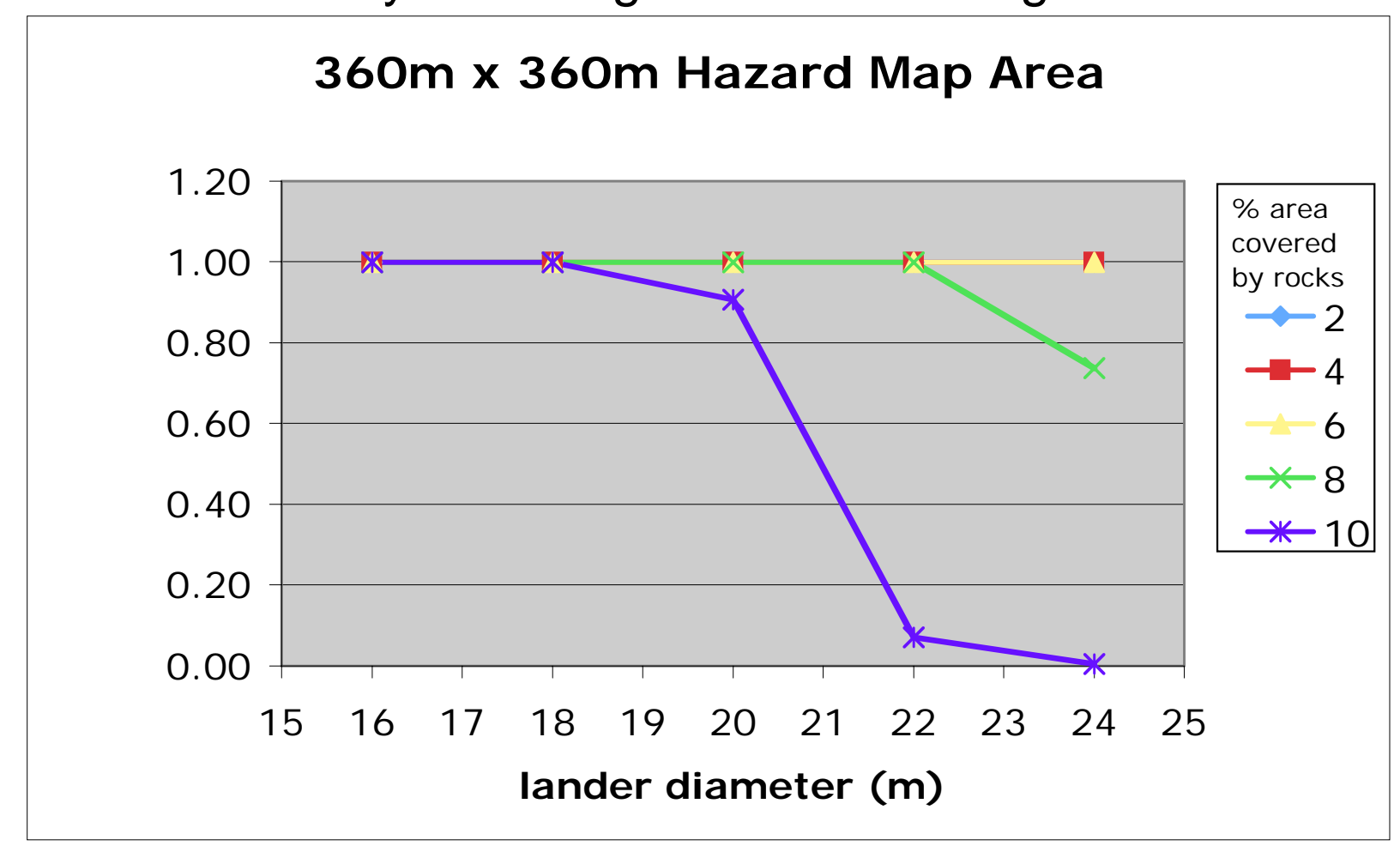

- Only rocks considered based on published statistical rock distribution models

- $30 \mathrm{~cm}$ rock is considered a hazard

Monte Carlo Analysis by Dr. Andrew Johnson, JPL, 2007 


\section{LANDER TOLERANCE}

Autonomous Landing Hazard Avoidance Technology (ALHAT)
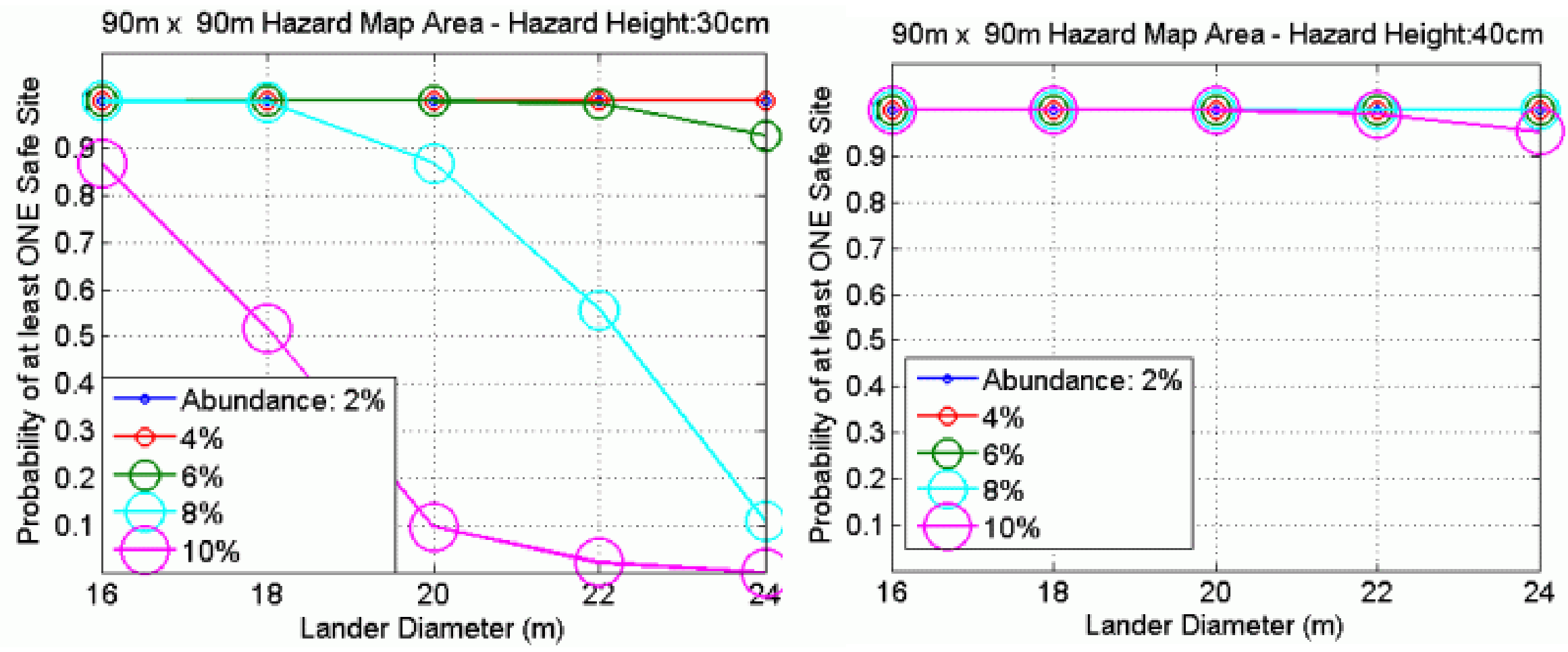

Based on published statistical rock distribution models

Monte Carlo Analysis by Dr. Andrew Johnson, JPL, 2007 


\section{HDA TERRAIN SENSORS}

- Human eye

- Requires some light

- Unclear about the ability to define hazards and hazard elevations, particularly holes and slopes, with significant shadows and contrast problems like expected at Shackleton Crater

- Current analysis and research underway to better understand this capability

- Infrared sensing systems and low light enhancing systems

- Usefulness of these systems at the lunar surface is not well understood and there exist some disagreements

- Current analysis and research underway to better understand this capability 


\section{HDA TERRAIN SENSORS}

- Optical cameras

- Requires some light

- Provides 2-D image with adequate light

- Limited ability to define hazards and hazard elevations, particularly holes and slopes, with lighting conditions expected at Shackleton Crater

- With image processing for comparison with preflight maps of hazards

- If preflight maps of hazards are good enough this approach could be used for hazard relative navigation

- Requires significant data processing

- Data available for display in near real-time (few seconds)

- Stereo cameras

- Requires large baseline

- Not considered a viable option for real-time imaging from altitudes between $500 \mathrm{~m}$ and $1000 \mathrm{~m}$ 


\section{HDA TERRAIN SENSORS}

- Flash LIDAR

- Digital camera which carries its own light source and able to measure time of flight for light striking each pixel (thus elevation data)

- Data independent of surface lighting conditions

- Provides Digital Elevation Map of the surface thus identifying rocks, holes and slopes

- Data utilized for hazard relative navigation to safe landing site

- Requires significant data processing

- Data available in near real-time (few seconds)

- Scanning LIDAR

- Similar to flash LIDAR except uses scanning beam

- Technology slightly more mature than flash

- Time required for data collection and processing is higher than for flash

- More moving parts than flash 


\section{HDA SENSOR PERFORMANCE}

- Must support landing without surface or orbiting navigation aids (supports sortie and first down outpost)

- Sensors required to provide real-time surface hazard elevation information

- Factors effecting sensor performance

- Range at which sensor capability provides required hazard resolution

- Higher the better

- Allows time to analyze, divert and fly efficiently to safe site

- Time required to obtain hazard information and define safe landing site

- Depends on range from target site data and deceleration rate

- Approximately 1.0 to 1.5 minutes to collect the data, process it, do analysis to pick safe site and divert to the safe site 


\section{CREWED LANDINGS}

- ALHAT has been challenged to analyze a crewed mission to a landing site with polar lighting conditions with no navigation aids and utilizing the crew's capabilities to maximum extent possible to determine the "minimum" suite of navigation/sensor equipment to land safely on the lunar surface.

- Based on the ALHAT Team current research and analysis knowledge, we believe a LIDAR provides the best option to meet safe landing requirements

- Considered to be a good sensor for determining real-time elevation information

- Additional development required to improve capability and resolution at higher altitudes and improve efficiency, robustness, reliability and space qualification

- Real-time LIDAR data requires high volume and high speed processing

- As our knowledge base increases it is possible that we will find other systems that can meet safe landing requirements

- Considering options such as infrared sensors, flood lights with crew capability, cameras, etc. 


\section{SUMMARY}

- The trade space of the ALHAT technology development includes a number of options

- Plan to have considerable analysis and test data by the end of FY08 that will narrow down the trade space and better define what is required for safe landing anywhere on the moon

- ALHAT is investigating human capability and aids to help humans detect hazards is part of the ALHAT development

- Important to continue to develop promising low TRL technologies that assure safe landing for crewed and autonomous vehicles for outpost and sortie missions 